\title{
Clostridium difficile (Cd) in Shelter Dogs: Rationale for Screening of Pets Slated for Adoption
}

\author{
Abdelrahman Mohamed ${ }^{1}$, Gopal P. Reddy ${ }^{1}$, Temesgen Samuel ${ }^{1}$, \\ Mahmoud Mansour ${ }^{2}$, Abdela Woubit ${ }^{1 *}$ \\ ${ }^{1}$ Department of Pathobiology, College of Veterinary Medicine Nursing and Allied Health, Tuskegee University, Tuskegee, AL 36088, USA \\ ${ }^{2}$ Department of Anatomy, Physiology and Pharmacology, College of Veterinary Medicine, Auburn University, Auburn, AL 36849, USA
}

\begin{abstract}
Received: March 03, 2016; Accepted: April 05, 2016; Published: April 16, 2016
*Corresponding author: Woubit Abdela, Department of Pathobiology, College of Veterinary Medicine Nursing and Allied Health, Tuskegee University, Tuskegee, AL 36088, USA, Telephone +334-727 8278; Fax +334-724-4277; E-mail: wabdela@mytu.tuskegee.edu
\end{abstract}

\begin{abstract}
Clostridium difficile (Cd)-associated disease is an important nosocomial bacterial infection. However, non-hospital-associated infections have been recently reported. We investigated the occurrence of Cd in apparently healthy household and in sheltered dogs. A total of 197 rectal swab samples were collected from male and female dogs from two shelters and from private homes in two counties in Alabama, USA. Eight out of the 197 samples (4.1\%) were confirmed positive for $\mathrm{Cd}$ by latex agglutination test. Ribotyping and toxin gene characterization by PCR showed five serotypes (I-V) with 3 isolates carrying $\mathrm{A}+\mathrm{B}+$ genes and other 3 carrying $\mathrm{A}-\mathrm{B}+$ genes. However, further analysis of the 6 toxin positive isolates with ELISA showed only 3 isolates $50 \%(3 / 6)$ as positive. All Cd positive samples were from sheltered dogs. Because sheltered dogs could be potential sources for Cd infections, we recommend screening of sheltered dogs for toxigenic $\mathrm{Cd}$ as part of a routine procedure for adoption.
\end{abstract}

Keywords: Clostridium difficile; Ribotyping; Toxin genes; Sheltered dogs

\section{Introduction}

Clostridium difficile (Cd) is a gram-positive spore-forming anaerobic bacillus that causes gastroenteritis in many animals as well as humans [1-7]. The disease in humans is well studied and infection ranges from mild or moderate diarrhea to severe life threatening Pseudomembranous Colitis (PMC) [8]. In the United States, recent finding shows an increase in the incidence of both community-acquired and hospital-acquired Cd infections [9]. Besides toxigenicity, prevalence of hyper-virulent multi-drug resistant strains is on the rise [10]. In humans, the presence of toxigenic $\mathrm{Cd}$ in the gut can be associated with disease in immunecompromised patients [11], the elderly, and/or in people on long-term antibiotic treatment.

Infection with $\mathrm{Cd}$ causes clinical disease by two potent exotoxins, toxin $\mathrm{A}(t c d \mathrm{~A})$ enterotoxin and toxin $\mathrm{B}(t c d \mathrm{~B})$ cytotoxin both located in the pathogenicity locus of this bacterium [12]. This locus also has three accessory genes, $t c d \mathrm{C}, t c d \mathrm{R}$ and $t c d \mathrm{E}$. Recently, Cd strains that are toxin A negative and toxin B positive were implicated in clinical Cd infections [13]. Clinical infection with Cd was originally associated with prior use of antibiotics [14], however, active community-associated Cd infections that are not linked to antibiotic therapy or hospitalization have recently emerged, suggesting risk factors other than antibiotics use in Cd infections $[15,16]$. In addition, toxigenic Cd strains were isolated from large swaths of potential sources that include pet animals [1], human food, food animals such as cattle, swine, and horses [2,3,6,17], and from the environment [18].

Companion animals could play critical role in communityacquired Cd infections if they harbor any of the hyper-virulent antimicrobial-resistant toxigenic Cd strains [19]. Dogs in particular, given their intimacy with humans, could be potential reservoirs for $\mathrm{Cd}$ infections, especially in the elderly, immunecompromised, and hospitalized individuals [20]. Prevalence of $\mathrm{Cd}$ in sick and healthy dogs and their implication as a possible reservoir for community-acquired $\mathrm{Cd}$ infections was previously suggested [7,21-23]. Studies on the prevalence and characterization of $\mathrm{Cd}$ in sheltered dogs are limited; one study was conducted overseas, Germany, [24] while the other study was performed on dogs housed in temporary shelters at the University of California Veterinary Medical Hospital, Davis [21].

The objective of this work was to investigate if sheltered and household dogs could be potential sources for $\mathrm{Cd}$ acquisition. Here, we compared the prevalence of $\mathrm{Cd}$ in healthy household dogs versus dogs kept in shelters for long- term/ long-term sheltered dogs. We also examined if any of the Cd isolates in dogs bore toxin associated genes.

\section{Materials and Methods}

\section{Study area, gender, age, and number of dogs}

Two groups of dogs, from shelters in two counties in the state of Alabama (Lee and Russell counties, AL, USA), and a third group from household dogs, in one of the above counties, were examined. Of the total 197 samples investigated, 105 were obtained from males and 92 were from females. Among the 197 dogs, Group A consisted of 70 male and female dogs (35 from each gender). These dogs were from first Humane Shelter in Lee County. Group B consisted of 60 total dogs, 36 males and 24 
females from a second Humane Shelter in Russell County. Group C consisted of 67 dogs, 34 males and 33 females from household dogs in the same county as Group A. The median age for the study of household dog animals was 6.6 years with a mean of 4.3 years. The household dogs did not receive any antibiotic treatment or visited a veterinary hospital in the past two months prior to the study.

\section{Sample collection and Cd culture}

A total of 197 anaerobic rectal charcoal swabs (Thermo Fisher Scientific Remel Products, Lenexa, KS) were obtained as part of a survey. They were immediately stored at $4^{\circ} \mathrm{C}$. Following collection swabs were then transported to the laboratory and processed within 2 hours after collection.

Charcoal swabs were inoculated into pre-reduced Cycloserine-Cefoxitin-Fructose Broth (CCFB) tubes with Cd selective supplement (Thermo Fisher Scientific Remel Products, Lenexa, KS) and 0.1\% sodium taurocholate (Biosynth International, Itasca, IL). The CCFB broth was prepared in the laboratory as described by [22]. The CCFB tubes were incubated at $37^{\circ} \mathrm{C}$ for 48 hours anaerobically using Gaspak anaerobic chamber (Thermo Fisher Scientific Remel Products, Lenexa, KS). Alcohol shock was performed as described elsewhere [23]. Samples were then centrifuged at 14,000 rpm for 10 minutes, supernatant was discarded and aliquots were streaked onto prereduced Cycloserine-Cefoxitin-Fructose Agar (CCFA) 9 Applied Biosystems, Foster City, CA) with Cd selective supplement and 7\% laked horse hemolyzed blood and vitamin K-hemin (Thermo Fisher Scientific Remel Products, Lenexa, KS). The agar culture plates were incubated anaerobically at $37^{\circ} \mathrm{C}$ for 48 hours. Control medium was inoculated with ATCC 9689 strain as positive control.

\section{Identification of (Cd) isolate}

Presumptive Cd growths were identified by colony morphology, gram stain appearance, horse manure smell, and L-proline aminopeptidase test (Thermo Fisher Scientific Remel Products, Lenexa, KS). Confirmation was performed using Clostridium difficile latex agglutination test (Microgen Bioproducts, Surrey, UK). Confirmed isolates were then subcultured on Brain Heart Infusion agar (BD Biosciences, Sparks, MD), supplemented with 5\% horse blood and incubated anaerobically at $37^{\circ} \mathrm{C}$ for 48 hours. Presence of toxins secreting isolates was confirmed with ELISA, $\mathrm{X} /$ pect Clostridium difficile Toxin A/B test kit (Thermo Fisher Scientific Remel Products, Lenexa, KS).

\section{DNA extraction}

DNA was extracted from 3-5 pure colonies and re-suspended in PrepMan ${ }^{\circledR}$ Ultra Sample Preparation kit following the manufacturer procedure (Applied Biosystems, Foster City, CA).

\section{Primers design for PCR ribotyping and toxin gene analysis}

The completed genome sequence data of Cd strain 630 (Accession numbers NC_008226 for plasmid and NC_009089 for reference genome) was retrieved from the NCBI (http:// www.ncbi.nlm.nih.gov/genomes/lproks.cgi) microbial genome sequencing database to V-NTI Advanced-11 (Life Technologies, Grand Island, NY) for the design and modification of primers. Primers 16S-F-m and 23S-R-m2 for PCR-ribotyping were designed with modification from previous work [25]. The ribotyping forward primer in this study was spaced only 45 bp upstream of the published forward primer (see Table 3) and therefore targeted the same locus. We could not locate the published reverse primer sequence on the genome strain 630 . The primers we used yielded band patterns between 332-664 base pair (bp). These primers were then analyzed in-silico at (http://insilico.ehu.es/PCR/) for the number of fragments and clear band resolution. Likewise, primers for toxin and associated genes $t c d \mathrm{~A}, t c d \mathrm{~B}, t c d \mathrm{C}, t c d \mathrm{D}$ and $t c d \mathrm{E}$ were designed from strain 630 that contains the toxin operon. The primers for toxins were designed to provide $600,500,400,300$, and $200 \mathrm{bp}$, respectively (Table 3). Specificities of these primers were confirmed using BLAST (http://www.ncbi.nlm.nih.gov/blast/) \& in-silico analysis (http://insilico.ehu.es/PCR/). Furthermore, V-NTI Advanced-11 software was used for testing of these primers including oligodimer, hair-loop formation and similar melting temperatures. Only primers showing the most specificity to the target sequences were ordered from Integrated DNA Technologies (IDT, Coralville, IA). Finally, presence of toxin proteins were analyzed using X/ pect ELISA kit (Thermo Fisher Scientific Remel Products, Lenexa, KS). PCR amplification profiles and toxin analysis were compared with reference type strain Cd ATCC-9689 (American Type Culture Collection, Manassas, VA).

All PCR reactions were set up on an isolated PCR station (AirClean Systems, Raleigh, NC) that was UV-treated daily, and after each use. Pentaplex PCR for toxin gene analysis and PCR for ribotyping were performed in $50 \mu \mathrm{l}$ final volume containing $0.2 \mu \mathrm{M}$ of forward and reverse primers, $25 \mu \mathrm{l}$ of Pwo Master mix containing $1.25 \mathrm{U}$ of Pwo enzyme, $2 \mathrm{mM} \mathrm{MgCl}_{2}$ and $0.2 \mathrm{mM}$ dNTPs (Roche Diagnostics, Mannheim, Germany). The PCR amplification program consisted of $10 \mathrm{~min}$ at $95^{\circ} \mathrm{C}$, followed by 30 cycles of 15 seconds at $95^{\circ} \mathrm{C}, 15$ seconds at $60^{\circ} \mathrm{C}$, and 60 seconds at $72^{\circ} \mathrm{C}$ using Master cycler pro (Eppendorf, Humburg, Germany). Presence of bands was analyzed using gel electrophoresis on $2.5 \%$ of agarose (NuSieve 3:1 AGAROSE, Lonza, Houston, TX, USA).

\section{Data analysis}

Data were analyzed by logistic regression analysis as described previously [21].

\section{Results}

In the present study $\mathrm{Cd}$ was isolated from apparently healthy dogs at prevalence rates of $4.1 \%$ in the study area. Of the 197 total rectal swabs cultured in CCF selective medium, 13 showed positive results with pro test but only 8 were confirmed positive for $\mathrm{Cd}$ with latex agglutination kit. $\mathrm{Cd}$ was detected only in samples from sheltered dogs (groups A and B) none was detected in household dogs (group C). In sheltered dogs, Cd was detected in $8.6 \%,(6 / 70)$ sheltered dogs in group $A$, and in $3.3 \%,(2 / 60)$ sheltered dogs in group B (Table 1). 
PCR ribotyping of Cd isolates revealed five types: type I three samples $(258,298,311)$, type II one sample (269), type III one sample (289), type IV two samples $(304,262)$ and type V one sample (316) (Figure 1). Two of the isolates (lanes 9 and 11) were similar to the reference strain Cd ATCC 9689. Ribotypes I, II \& III were all from dogs in shelter A, ribotype IV was shared between group A and B one sample for each. Ribotype $V$ was detected in one sample from group B.

Analysis of the toxin associated genes revealed presence of the highly potent toxins A and B in $75 \%$ of all the isolates identified (6/8), with the exception of 289 and 316. In addition sample 316 has a visual weak band of toxin B gene. Of the 70 dogs from group A, 5 out of 6 of these dogs was harboring toxigenic genes $(62.5 \%), 2$ were $\mathrm{A}+\mathrm{B}+$ variant and 3 were $\mathrm{A}-\mathrm{B}+$ variant (Table 2). Of the 60 dogs from group B, 2 was $C d$ positive but only $1(12.5 \%)$ carries the toxic genes of $\mathrm{Cd}, \mathrm{A}+\mathrm{B}+$ variant (Table 2). One isolate $A+B+$ from group $A$ and another $A+B+$ variant from group $B$ showed presence of $c d t R$ gene similar to positive control (Figure 2). Of the $8 \mathrm{Cd}$ positive isolates, three samples were positive for toxin production (A and/or B) when analyzed using X/pect ELISA kit (Remel) yielding a $37.5 \%$ (3/8) toxin producing Cd variants, (2 from group A and 1 from group B). Of the 67 household dogs in group $C$, none was positive or shedding Cd (Table 2). The sheltered dogs were all adult dogs but their age was unknown and thus could not be evaluated for age/ carriage analysis. Although the age factor in group C dogs (median of 6.6 years) was known, it has no bearing on Cd colonization since the entire group tested was negative.

Analysis of the confirmed $\mathrm{Cd}$ colonization data based on gender, irrespective of the study area, showed a higher prevalence of $\mathrm{Cd}$ in females compared to males. Of the 8 samples that were positive in group A and B, 5 were from females (62.5\%) compared to $3(37.5 \%)$ that were from males. Within the study groups, the prevalence of $\mathrm{Cd}$ in male dogs was the highest in Group B (5.6\%) compared to (2.9\%) in Group A. In contrast, the Cd prevalence in female dogs was $(0 \%)$ in Group B compared to $(14.3 \%)$ in Group A (Table 1). Overall the carriage rate of Cd was significantly higher for females when compared to male dogs (odds ratio (OR) $=1.9595,95 \%$ Confidence Interval $(\mathrm{CI})=0.453$ to 8.4759$)$.

Table 1: Prevalence of Cd in the study area.

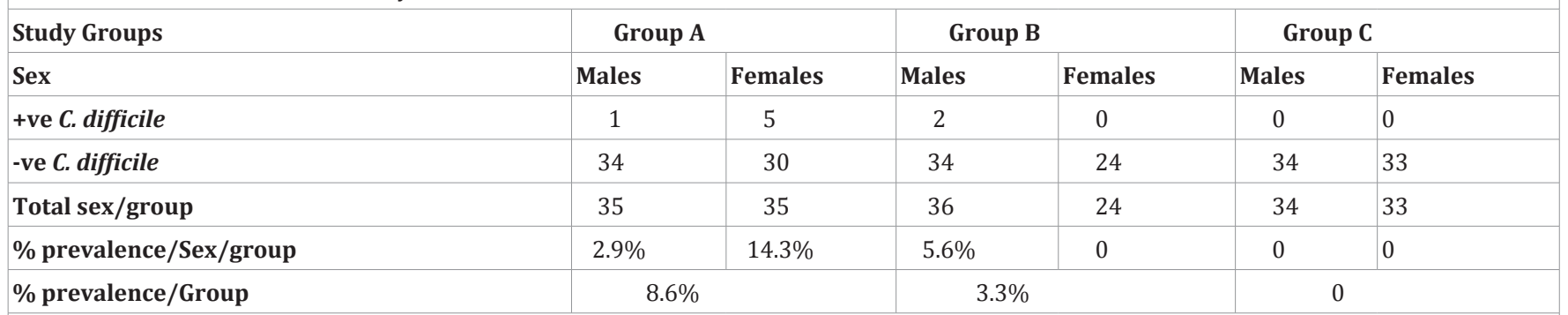

Prevalence of Cd in the study area. Group A and B are sheltered dogs, while Group C is privately owned dogs.

Overall, group A has the highest prevalence while group C has none.

The carriage rate of $\mathrm{Cd}$ was significantly higher in female dogs compared to male dogs (odds ratio $(\mathrm{OR})=1.9595,95 \%$ confidence interval $(\mathrm{CI})=$ 0.453 to 8.4759 ).

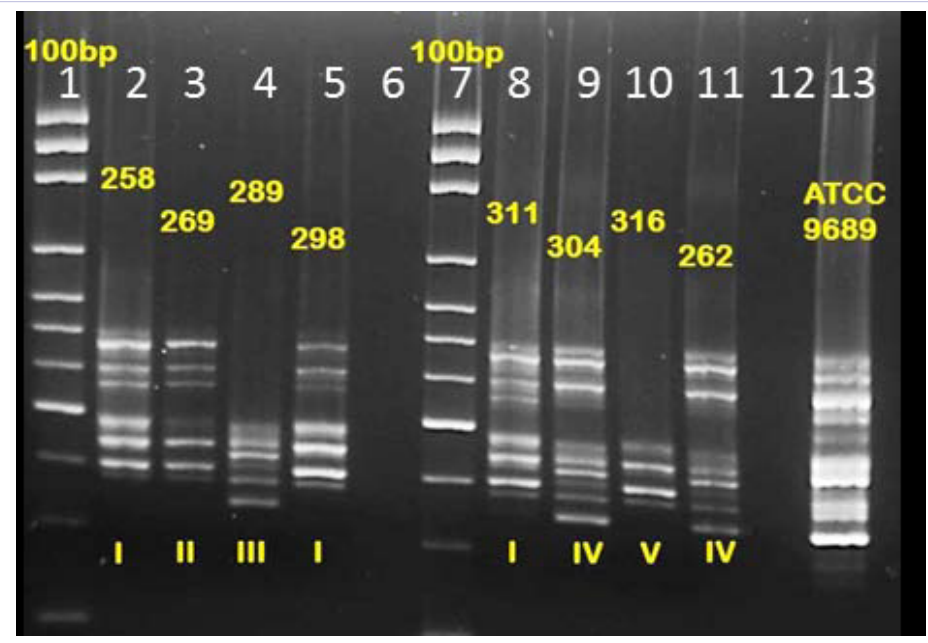

Figure 1: Gel electrophoresis of Clostridium difficile (Cd) ribotyping. Among $8 \mathrm{Cd}$ positive isolates, 5 different ribotypes (I-V) were found based on their gel electrophoresis profiles. These profiles include: type I (samples 258, 298, and 311) in lanes 2, 5, 8, respectively, type II (sample 269) in lane 3, type III (sample 289) in lane 4, type IV (sample 304, 262) in lanes 9 and 11; and type V (sample 316) in lane 10; 100 bp DNA markers were included in lanes 1 and 7. ATCC 9689 positive control was in lane 13. 
Table 2: Toxin characterization of $\mathrm{Cd}$ in the study area.

\begin{tabular}{|l|c|c|c|}
\hline Study Groups & Group A & Group B & Group C \\
\hline A+/B+ isolates & 2 & 1 & 0 \\
\hline A-/B+ isolates & 3 & 0 & 0 \\
\hline A-/B- isolates & 1 & 1 & 0 \\
\hline A/B Eliza positive isolates & 2 & 1 & 0 \\
\hline
\end{tabular}

Toxin characterization in the study area. 5 of toxigenic $\mathrm{Cd}$ variants from group A were $\mathrm{A}+$ and/or $\mathrm{B}+(62.5 \%)$ toxigenic strain while only one toxic variant was from group B (12.5\%), two ELISA positive variants were from group A and one from group B.

Table 3: Primers used for ribotyping and toxinotyping.

\begin{tabular}{|c|c|c|c|}
\hline Name & Size amplified & Primer sequence & Origin \\
\hline $16 S-F$ & & 5'-CTGGGGTGAAGTCGTAACAAGG-3' & 0, Neill, et al. [25] 1441-1464 \\
\hline 23S-R & & 5'-GCGCCCTTTGTAGCTTGACC-3' & $”$ \\
\hline $16 \mathrm{~S}-\mathrm{F}-\mathrm{m}$ & $332-664 \mathrm{bp}$ & 5'-CCGAAGCCGATTATCTAACCT-3' & $\begin{array}{l}\text { This study } \\
1384-1404\end{array}$ \\
\hline 23S-R-m2 & & 5'-CTCCTAGTGCCAAGGCATCC-3' & $"$ \\
\hline ToxinA-F & $600 \mathrm{bp}$ & GGATGGAATTTATATATGATAGAC & $"$ \\
\hline ToxinA-R & & CTGCCTAAAGCGAAAGCTATTT & $"$ \\
\hline ToxinB-F & $500 \mathrm{bp}$ & AGCTCAAAGAGAAGAAAATCCTG & $"$ \\
\hline ToxinB-R & & TTCACAGAAATTAGCCCTTGAT & $"$ \\
\hline ToxinC-F & $400 \mathrm{bp}$ & ATTTCCACCCATAGTTGATTCA & $"$ \\
\hline ToxinC-R & & ACCATGAGGAGGTCATTTCTAA & $"$ \\
\hline ToxinR-D-F & $300 \mathrm{bp}$ & GAGTTTTACATTATGAAGAGGGAGA & $”$ \\
\hline ToxinR-D-R & & CTATTTTTAGCCTTATTAACAG & $"$ \\
\hline ToxinE-R & $200 \mathrm{bp}$ & TCTAGTTTTGGAATAGATGGAGGA & $"$ \\
\hline ToxinE-F & & CTTAGCATTCATTTCATCTGTC & $”$ \\
\hline
\end{tabular}

Primers used in the study. Ribotyping primers used (16SF) was spaced only 45bp upstream of a previously published one (O’Neill, et al. [25]).

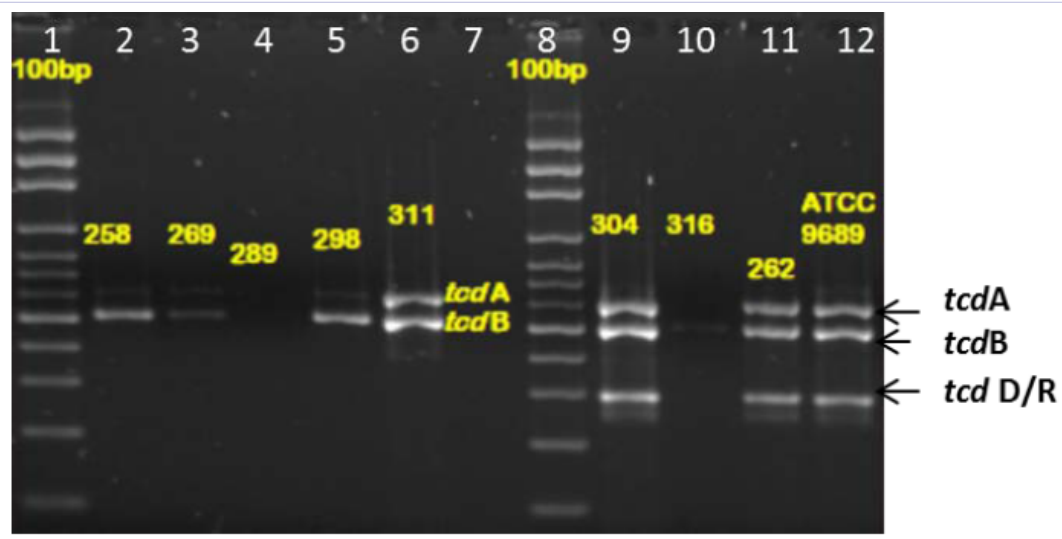

Figure 2: Gel electrophoresis of Clostridium difficile $(\mathrm{Cd}) t c d \mathrm{~A}, t c d \mathrm{~B}, t c d \mathrm{D} / \mathrm{R}$ toxin genes detected by PCR. Genes for Toxin A and B were detected in all of the isolates (lanes 2-6 and 9-11) except in sample 289 (lane 4) and weak signal in sample 316 (lane 10). Besides the two major toxin genes A/B, isolates 304 (lane 9) and 262 (lane 11) possess genes tcdD/R like the type strain Clostridium difficile ATCC 9689.

\section{Discussion}

The objective of this study was to investigate the prevalence of Cd in household dogs and long-term sheltered dogs. Cd was isolated from asymptomatic dogs with prevalence rates of $4.1 \%$, $8 / 197$. Three observations can be surmised from the difference in
Cd prevalence calculated from the Pro test and latex agglutination results. First, the latex agglutination is more specific than the Pro test as it excluded 5 samples that were initially positive in the Pro test. Second, out of the 8 positive isolates, $75 \%$ were carrying toxin genes (6/8). Third, as a matter of practical importance, 
Cd detected by the Pro test should always be further confirmed by latex agglutination test and/or PCR analysis for gluD and tpi genes.

Interestingly, of the three study groups, the Cd prevalence was highest in sheltered dogs from group A at $8.6 \%$ followed by group B with 3.3\% prevalence. None of the household dogs, group $\mathrm{C}$ were positive for $\mathrm{Cd}$, suggesting $0 \%$ prevalence in this group.

The differences in Cd prevalence between our three study groups could be due to differences in the housing environment and/ or management conditions. Given the association of Cd with the use of broad spectrum antibiotics, it is possible that the lack of Cd colonization in household dogs is due to absence of antecedent antibiotic exposure in this group and limited contact with many dogs as the case of sheltered dogs. Indeed in our communications with the owners of household dogs, none of the dogs in this group was treated with antibiotics in the last three months.

Although the use of antibiotics in sheltered dogs is common, the lack of information on their clinical history does not allow us to suggest a direct link. Stress factor cannot be ignored as sheltered dogs lost their caring owners and comfortable lodgings where they end up living in different crowded houses. Another possibility is that sheltered dogs could acquire Cd from other dogs in the shelter. It is possible that acquirement of $\mathrm{Cd}$ from the shelter is more likely to happen as the spores of $\mathrm{Cd}$ can persist for long time from previous spore-shedder dogs.

The lack of Cd carriage in household dogs is not surprising and generally reflects the lower carriage rate of both toxigenic and non-toxigenic $\mathrm{Cd}$ previously reported in healthy dogs in the community [7]. A previous study that tested Cd carriage in multiple species, including 52 household dogs, found $21 \%$ prevalence (11/52) all are non- cytotoxigenic $\mathrm{Cd}$, with only one dog with a cytotoxigenic $\mathrm{Cd}$ strain. This suggests that $\mathrm{Cd}$ carriage in household pets is insignificant and perhaps is comparable to the lack of Cd pathogenicity in human infants [26].

Previous studies that evaluated Cd infection in normal versus diarrheic dogs reported Cd prevalence that varied between $4 \%$ [7] to $7 \%$ [4]. These figures are comparable to our findings of $4.1 \%$ prevalence in shelter dogs.

Two previous studies evaluated Cd prevalence in normal dogs housed in shelters, of which one was conducted in Europe. In the first study (conducted in Germany) Cd was isolated from 9 out of $165(5.5 \%)$ dog samples and 5 out of $135(3.7 \%)$ cat samples. Five PCR ribotypes $(010,014 / 020,039,045$, SLO 066) were identified [24]. The second study was performed at the University of California (Davis) School of Veterinary Medicine where stool specimens from 152 dogs (in- and outpatients) were analyzed for the presence of $\mathrm{Cd}$. An additional 42 stool specimens from dogs described as "recently" housed at local animal shelters were also examined [21]. Although Cd was isolated from the feces of 28 of the veterinary hospital patients $(18.4 \%)$ with 14 of the isolates $(50.0 \%)$ described as toxigenic, none was isolated from the 42 sheltered dogs. The shelter conditions in the University of California were described by the authors as temporary, thus different from the long-term dogs shelters used in our study. However, the $5.5 \%$ Cd prevalence reported in the German study is akin to our findings.

\section{Conclusion}

Our study identifies sheltered dogs as a potential source for toxinogenic Cd; our study suggests that dogs housed in shelters could be reservoirs for $\mathrm{Cd}$ transmission to humans. Incorporation of customized tests for $\mathrm{Cd}$ diagnosis in sheltered dogs is desirable especially when they are offered for adoption and the adoptee is an elderly individual and/ or someone with an immunecompromised health condition.

\section{Acknowledgements}

This work was supported by USDA/NIFA/ISE 2009-5116005482 (Dr. G. Reddy), MARC-5T34GM08 (Dr. Martinez), and NCFPD2007-ST-061-000003 (Dr. Woubit A). Authors would like to thank Dr. Magda Abo Samah and T. Collins for laboratory assistance, Veronica Simmons and Naree McCoy for the help with samples collection, Russell and Macon Counties Humane Societies and Caitlin Trebelhorn, Dr. Solomon Odemuyiwa and Toufic Nashar for reading the manuscript.

\section{References}

1. Clooten J, Kruth S, Arroyo L, Weese JS. Prevalence and risk factors for Clostridium difficile colonization in dogs and cats hospitalized in an intensive care unit. Veterinary Microbiology. 2008;129(1-2):209-214. doi: 10.1016/j.vetmic.2007.11.013.

2. Medina-Torres CE, Weese JS, Staempfli HR. Prevalence of Clostridium difficile in horses. Vet Microbiol. 2011;152:212-215. doi: 10.1016/j. vetmic.2011.04.012.

3. Thakur S, Putnam M, Fry PR, Abley M, Gebreyes WA. Prevalence of antimicrobial resistance and association with toxin genes in Clostridium difficile in commercial swine. Am J Vet Res 2010;71:11891194. doi: 10.2460/ajvr.71.10.1189.

4. Weese JS, Staempfli HR, Prescott JF, Kruth SA, Greenwood SJ, Weese HE. The Roles of Clostridium difficile and Enterotoxigenic Clostridium perfringens in Diarrhea in Dogs. J Vet Intern Med. 2001;15:374-378.

5. al-Barrak A, Embil J, Dyck B, Olekson K, Nicoll D, Alfa M, et al. An outbreak of toxin A negative, toxin B positive Clostridium difficileassociated diarrhea in a Canadian tertiary-care hospital. Can Commun Dis Rep. 1999;25:65-69.

6. Rodriguez-Palacios A, Stampfli HR, Duffield T, Peregrine AS, TrotzWilliams LA, Arroyo LG, Brazier JS, Weese JS. Clostridium difficile PCR ribotypes in calves, Canada. Emerg Infect Dis. 2006;12:1730-1736.

7. Wetterwik K-J, Trowald-Wigh G, Fernstrom L-L, Krovacek K. Clostridium difficile in faeces from healthy dogs and dogs with diarrhea. Acta Veterinaria Scandinavica. 2013;55:23.

8. Musher D, Logan N, Mehendiratta V, Melgarejo N, Garud S, Hamill R. Clostridium difficile colitis that fails conventional metronidazole therapy: response to nitazoxanide. J Antimicrob Chemother. 2007;59:705-710.

9. Khanna S, Pardi DS, Aronson SL, Kammer PP, Orenstein R, St Sauver JL, Harmsen WS, Zinsmeister AR. The epidemiology of communityacquired Clostridium difficile infection: a population-based study. Am J Gastroenterol 2012;107:89-95. doi: 10.1038/ajg.2011.398. 
10. Valiente E, Dawson LF, Cairns MD, Stabler RA, Wren BW. Emergence of new PCR ribotypes from the hypervirulent Clostridium difficile 027 lineage. J Med microbiol. 2012;61:49-56. doi: 10.1099/jmm.0.0361940 .

11. Bruce D, Ritchie C, Jennings LC, Lynn KL, Bailey RR, Cook HB Clostridium difficile-associated colitis: cross infection in predisposed patients with renal failure. N Z Med J. 1982;95:265-267.

12. Voth DE, Ballard JD. Clostridium difficile Toxins: Mechanism of Action and Role in Disease. Clin Microbiol Rev. 2005;18(2):247-263.

13. Elliott B, Squire MM, Thean S, Chang BJ, Brazier JS, Rupnik M, et al. New types of toxin A-negative, toxin B-positive strains among clinical isolates of Clostridium difficile in Australia. J Med Microbiol. 2011;60(Pt 8): 1108-11. doi: 10.1099/jmm.0.031062-0.

14. Deshpande A, Pasupuleti V, Thota P, Pant C, Rolston DDK, Sferra TJ, et al. Community-associated Clostridium difficile infection and antibiotics: a meta-analysis. J Antimicrob Chemother. 2013;68(9):1951-61. doi: $10.1093 / j a c / d k t 129$.

15. Khanna S, Baddour LM, Huskins WC, Kammer PP, Faubion WA, Zinsmeister AR, Harmsen WS, Pardi DS. The epidemiology of Clostridium difficile infection in children: a population-based study. Clin Infect Dis. 2013;56(10):1401-6. doi: 10.1093/cid/cit075.

16. Chitnis AS, Holzbauer SM, Belflower RM, Winston LG, Bamberg WM, Lyons C, et al. Epidemiology of community-associated clostridium difficile infection, 2009 through 2011. JAMA Intern Med. 2013;173(14):1359-67. doi: 10.1001/jamainternmed.2013.7056.

17. Bakri MM, Brown DJ, Butcher JP, Sutherland AD. Clostridium difficile in ready-to-eat salads, Scotland. Emerg Infect Dis. 2009;15:817-818.

18. Weese JS, Finley R, Reid-Smith RR, Janecko N, Rousseau J. Evaluation of Clostridium difficile in dogs and the household environment. Epidemiol Infect. 2010;138:1100-1104.
19. Borriello S, Honour P, Turner T, Barclay F. Household pets as a potential reservoir for Clostridium difficile infection. J Clin Pathol.1983;36:84-87.

20. Lefebvre SL, Waltner-Toews D, Peregrine AS, Reid-Smith R, Hodge L, Arroyo LG, Weese JS. Prevalence of zoonotic agents in dogs visiting hospitalized people in Ontario: implications for infection control. J Hosp Infect 2006;62(4):458-466.

21. Struble AL, Tang YJ, Kass PH, Gumerlock PH, Madewell BR, Silva J, Jr. Fecal shedding of Clostridium difficile in dogs: a period prevalence survey in a veterinary medical teaching hospital. J Vet Diagn Invest. 1994;6(3):342-7.

22. Clabots CR, Johnson, S, Bettin KM, Mathie PA, Mulligan ME, Schaberg $\mathrm{DR}$, et al. Development of a rapid and efficient restriction endonuclease analysis typing system for Clostridium difficile and correlation with other typing systems. . J Clin Microbiol. 1993;31(7):1870-5.

23. Arroyo LG, Rousseau J, Willey BM, Low DE, Staempfli H, McGeer A, et al. Use of a selective enrichment broth to recover Clostridium difficile from stool swabs stored under different conditions. J Clin Microbiol. 2005;43(10):5341-3.

24. Schneeberg A, Rupnik M, Neubauer H, Seyboldt C. Prevalence and distribution of Clostridium difficile PCR ribotypes in cats and dogs from animal shelters in Thuringia, Germany. Anaerobe. 2012;18(5):484-8. doi: 10.1016/j.anaerobe.2012.08.002.

25. ONeill GL, Ogunsola FT, Brazier JS, Duerden BI. Modification of a PCR ribotyping method for application as a routine typing scheme for Clostridium difficile. Anaerobe. 1996;2:205-209.

26. Kato H, Kato N, Watanabe K, Ueno K, Ushijima H, Hashira S, Abe T. Application of typing by pulsed-field gel electrophoresis to the study of Clostridium difficile in a neonatal intensive care unit. J Clin Microbiol. 1994;32:2067-2070. 